\title{
4-Aminothiophenol Photodimerization Without
}

\section{Plasmons}

Ivano Alessandri $\mathbf{i}^{1,2,3 *}$

${ }^{1}$ Sustainable Chemistry and Materials Group, Department of Information Engineering, University of Brescia (Italy)

${ }^{2}$ INSTM, Consorzio Nazionale per la Scienza e Tecnologia dei Materiali, RU Brescia, via Branze

$$
\text { 38, } 25123 \text { Brescia (Italy) }
$$

${ }^{3}$ INO-CNR, RU Brescia, via Branze 43, 25123 Brescia (Italy)

e-mail: ivano.alessandri@unibs.it

\begin{abstract}
The photodimerization of 4-aminothiophenol (PATP) into 4,4'-dimercaptobenzene (DMAB) has been extensively utilized as a paradigm reaction to probe the role of surface plasmons in nanoparticlemediated light-driven processes. Over the past 25 years, a lively debate about the reaction mechanism involved several research groups. Plasmon-mediated generation of energetic (hot) electrons and holes has been invoked as the main driving-force, although plasmonic heating has recently gained attention. However, either model and their combinations are not sufficient to explain some experimental observations and appear, in some cases, contradictory. No matter the differences, there is a general, firm consensus about the presence of plasmonic nanoparticles (Ag or $\mathrm{Au}$ ), which has always been considered mandatory for triggering the photoconversion.

Here I report the first observation of the PATP-to-DMAB photoreaction in the absence of any plasmonic mediators. In particular, neither plasmonic heating nor charge transfer from hot carriers were exploited. The reaction was observed to occur with different kinetics on plasmon-free $\mathrm{TiO}_{2}$ nanoparticles, as well as on self-standing droplets. Confocal microRaman spectroscopy enabled to investigate the reaction progress in different plasmon-free contexts, either aerobic or anaerobic, suggesting a new interpretation of the photodimerization process, based on direct laser-induced activation of singlet oxygen species. These results provide new insights in light-driven redox processes, elucidating the role of sample morphology, light and oxygen.
\end{abstract}

Keywords: PAPT; DMAB; photodimerization; SERS; singlet oxygen; 
The photodimerization of 4-aminothiphenol (PATP) or 4-nitrothiophenol (PNTP) or into 4,4'dimercaptoazobenzene (DMAB) has attracted a lot of attention in the scientific community, as it has been a continuous source of vibrant debates. In a seminal paper, which dates back to 1994, Osawa et $a l$. reported the differences between the Surface Enhanced Raman spectra (SERS) of PATP adsorbed on silver nanoparticles and those of the bulk counterpart. ${ }^{1}$ For many years this remarkable difference has been ascribed to the so-called "chemical enhancement", resulting from charge transfer from metal to the adsorbed molecule. This effect was though to selectively enhance the non-totally symmetric (b2) modes of PAPT. Later on, other studies combining SERS and mass spectrometry demonstrated that the spectral differences are actually due to the formation of DMAB. ${ }^{2}$

Since then, the PAPT-to-DMAB photodimerization has been thoroughly investigated as a model reaction to test the capability of plasmonic systems to assist light-driven reactions. In this context, another debate on the reaction mechanism is still running. On one hand, hot carriers (electrons, in the case of PNTP photo-reduction or holes, in the case of PAPT photo-oxidation) have been invoked to assist photodimerization by either direct charge injection into the LUMO (hot electrons) or from the HOMO (hot holes) of the adsorbed molecules. ${ }^{3}$

However, recent studies pointed out the photothermal origin of the photoreaction. In particular, plasmonic heating has been indicated to play a key role, whereas pure thermal heating is not effective in driving photodimerization. ${ }^{4}$ This phenomenon is quite surprising, as the overall contribution of thermal energy should not be dependent on the heating source.

In spite of different models and several contradictions, all the studies agree with the mandatory presence of plasmonic nanoparticles and their key role in assisting the photoreaction. ${ }^{5}$ More generally, even when the reaction was run by applying an external voltage or by adding oxidants $\left(\mathrm{AgNO}_{3}, \mathrm{FeCl}_{3}\right.$, $\mathrm{H}_{2} \mathrm{O}_{2}$ ), metal nanoparticles have always been present. ${ }^{3,6}$

Here, I unexpectedly discovered that PAPT can undergo photodimerization in the absence of any metal mediators, by direct irradiation with a low power $633 \mathrm{~nm}$ laser $(1.96 \mathrm{eV})$. Surprisingly, past experiments reported the impossibility to directly convert PATP into DMAB by direct irradiation 
with visible lasers $(633,532,457 \mathrm{~nm}) .{ }^{7}$ However, in this work I observed that this reaction can be not only triggered, but also controlled, simply by modifying size and morphology of the sample under irradiation. A possible model, based on the laser-induced excitation of adsorbed molecular oxygen to singlet oxygen states is derived from confocal Raman microscopy experiments. Most of the unclear aspects of experimental data obtained for plasmonic systems can also be disentangled through this approach.

The first proof of direct, plasmon-free photoconversion of PAPT into DMAB was obtained unintentionally during the microRaman characterization of commercial $\mathrm{TiO}_{2}$ (Aeroxide® $\mathrm{P}-25$ ) powders previously soaked in a $10^{-4} \mathrm{M}$ PAPT ethanol solution for 30 minutes. Direct irradiation with a $633 \mathrm{~nm}$ laser focused through a 50X microscope objective (Numerical Aperture, N. A. : 0.5) with a power of $1.6 \mathrm{~mW} / \mu \mathrm{m}^{2}$, corresponding to $5 \times 10^{15}$ photons $/ \mu \mathrm{m}^{2} \mathrm{~s}$, resulted in a very fast $(<1 \mathrm{~s})$ degradation of the PAPT coating (see Supporting Information, Figure S1). By reducing the laser power to $0.16 \mathrm{~mW} / \mu \mathrm{m}^{2}\left(5 \times 10^{14}\right.$ photons $\left./ \mu \mathrm{m}^{2} \mathrm{~s}\right)$ the reaction can be controlled and its progress is shown in Figure 1.

We note that after $20 \mathrm{~s}$ new Raman modes appear at 1142, 1395 and $1443 \mathrm{~cm}^{-1}$ and progressively increase their intensity. According to previous studies, these signals are associated to the $\beta(\mathrm{CH})$ and $v(\mathrm{~N}=\mathrm{N})$ modes of DMAB ${ }^{8}$ In parallel, we observe a progressive increase of the spectral background as a function of irradiation time. After $140 \mathrm{~s}$ the background level becomes too high, leading to saturation of the spectral signal (see Figure S2). The Raman analysis of the same $\mathrm{TiO}_{2}$ powders soaked in ethanol and laser irradiated under the same conditions do not show any increase of the spectral background, which allows to exclude any contribution of the solvent to the background evolution (see Figure S3). Moreover, this control experiment reveals that the progressive increase of spectral background cannot be due to purely thermal effects. 


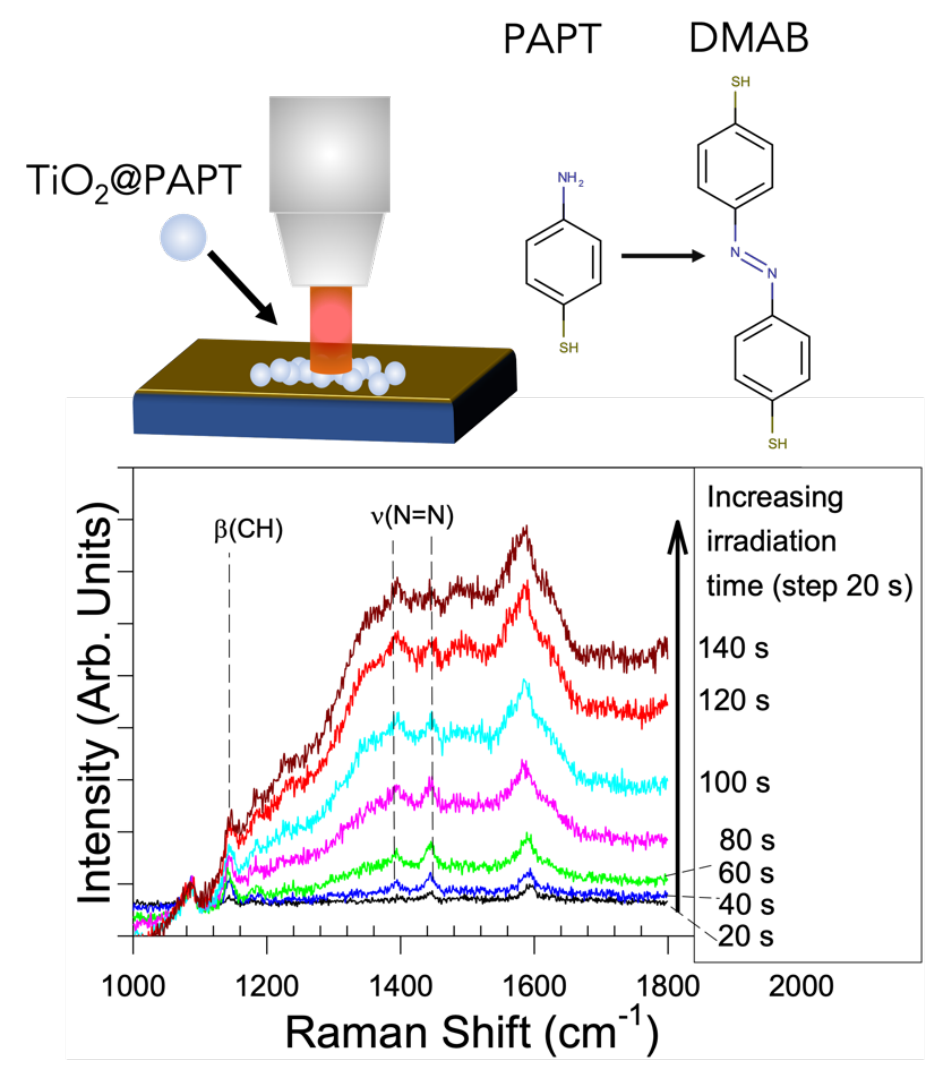

Figure 1. Evolution of PAPT photo-oxidation induced by laser irradiation at $633 \mathrm{~nm}$. The power was reduced to $0.16 \mathrm{~mW} / \mathrm{mm}^{2}$ to follow the formation of DMAB species, indicated by the $\beta(\mathrm{CH})$ and $v(\mathrm{~N}=\mathrm{N})$ Raman modes. Each spectrum was acquired for $20 \mathrm{~s}$ at the time interval indicated in the label.

Further experiments were carried out to gain more insight into the factors that trigger the reaction. For example, we observed that both the transformation of PAPT and related background onset occur even when the laser irradiation power was decreased to $16 \mu \mathrm{W} / \mu \mathrm{m}^{2}$ (see Figure $\mathrm{S} 4$ ), yet more slowly, indicating a direct dependence of reaction kinetics on the photon flux. The observation of photodimerization induced at $633 \mathrm{~nm}$ in the absence of any plasmonic mediators was unexpected, at least on the basis of the results reported in literature. For example, Zhang et al. did not observe any dimerization at $633 \mathrm{~nm}$ and concluded that the energy is not sufficient for triggering the azo-coupling reaction. ${ }^{7}$ Thus, the first hypothesis is that, in our case, $\mathrm{TiO}_{2}$ could be directly involved in assisting photodimerization. However, Figure S5 shows that the same PAPT solution deposited on planar $\mathrm{TiO}_{2}$ thin films does not undergo any photodimerization under the same conditions of the experiment reported in Figure 1, while by increasing the irradiation power to the pristine value of $1.6 \mathrm{~mW} / \mu \mathrm{m}^{2}$ 
the photo-oxidation process proceeds to completion. The rate of PAPT conversion on $\mathrm{SiO}_{2} / \mathrm{TiO}_{2}$ core/shell microbeads (T-rex) $)^{18}$ was in between that of P25 nanoparticles and planar $\mathrm{TiO}_{2}$ thin films . (Figure S6). These data suggest that the morphology of supports is more important than the chemical nature of the material in controlling the photooxidation kinetics.

A further confirmation in this regard is provided by the series of experiments shown in Figure 2 and S7-8, in which droplets of PAPT with a variety of different sizes were obtained by drying an aliquot (e.g. 5-10 $\mu \mathrm{L}$ ) of the same ethanol solution on either glass or silicon substrates in the absence of any $\mathrm{TiO}_{2}$ or other mediators. Surprisingly, the irradiation of these PAPT droplets at $633 \mathrm{~nm}$ gave different results depending on their size. Freshly deposited droplets, with size bigger than 20-30 $\mu$, were stable, even for prolonged (up to $35 \mathrm{~min}$.) irradiation at the maximum power $\left(1.6 \mathrm{~mW} / \mu \mathrm{m}^{2}\right)$. No DMAB spectral modes were observed and no evidence of any modification in droplet morphology was found from optical microscopy inspection (Figure 2a). However, when their size is reduced to the $1-5 \mu \mathrm{m}$ range, the droplets undergo a rapid oxidation that passes through dimerization and terminates with their complete carbonization, as shown in Raman spectra an optical microscope images (see Figure $2 \mathrm{~b}$ and $\mathrm{S} 7$ ). When the irradiation power was reduced to $0.16 \mathrm{~mW} / \mu \mathrm{m}^{2}$ the photooxidation kinetics was significantly slowed down and the progress of DMAB formation could be monitored with more detail (Figure S8). One can argue that the dependence of the reaction kinetics on the droplet size could be a proof of the photothermal nature of the process. In this regard, heat would be better dissipated over larger amount of sample, whereas small droplets could be more efficiently heated.

However, control experiments showed that the Raman spectra of small PAPT droplets remain unaltered when the sample is irradiated with a $785 \mathrm{~nm}$ laser, even though its power is remarkably higher $\left(260 \mathrm{~mW} / \mu \mathrm{m}^{2}\right.$, see Figure S9) than that of the $633 \mathrm{~nm}$ counterpart. This means that dimerization primarily depends on the wavelength of the exciting light source and pure thermal effects are not sufficient to drive dimerization. 


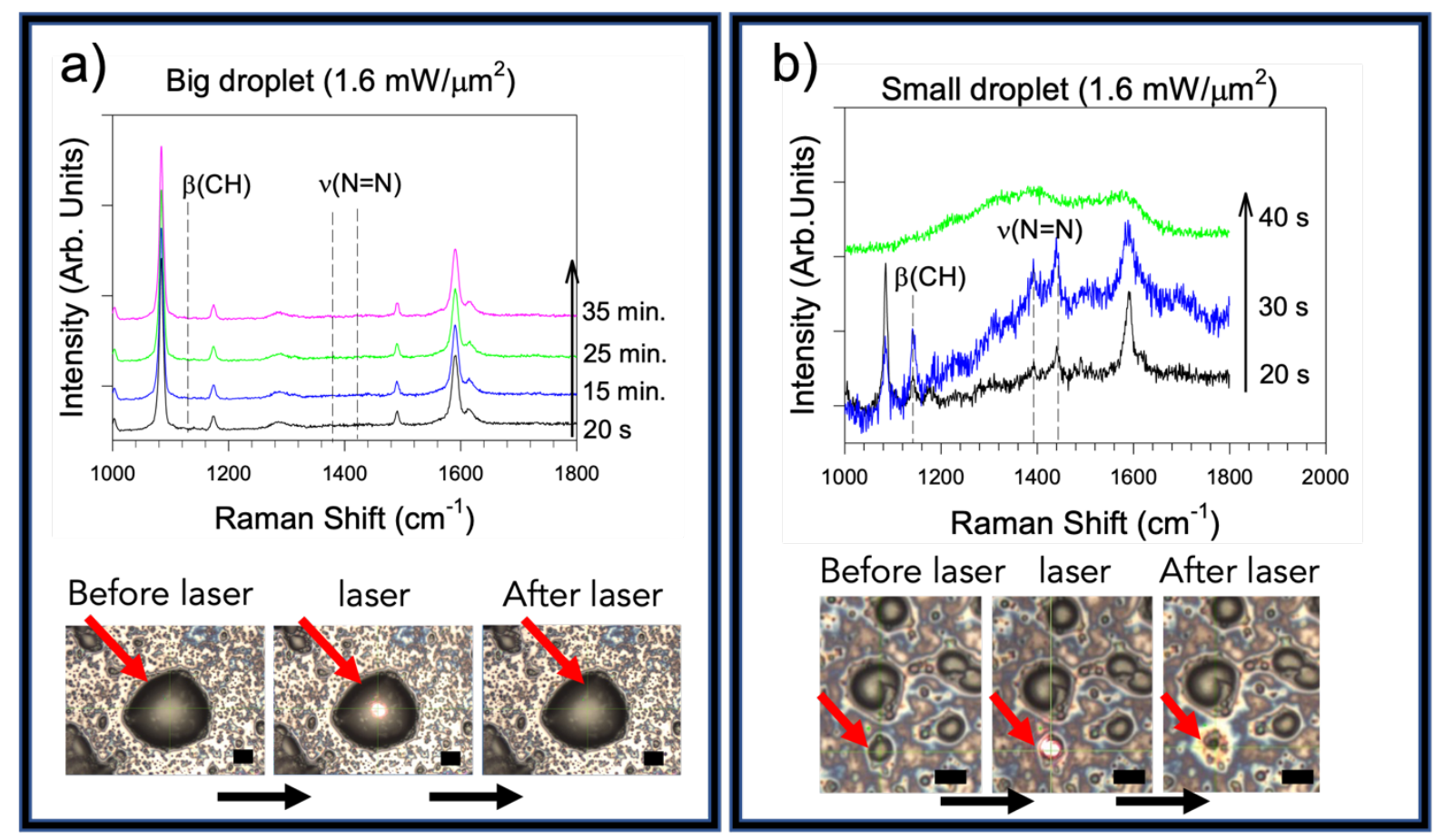

Figure 2. Spectral and morphological evolution of PAPT photo-oxidation induced by laser irradiation at $633 \mathrm{~nm}, 1.6 \mathrm{~mW} / \mu \mathrm{m}^{2}$ for a) big (i.e. $>30 \mu \mathrm{m}$ ) and small (i.e. $<5 \mu \mathrm{m}$ ) droplets. Each spectrum was acquired for $10 \mathrm{~s}$ at the intervals of time indicated in the labels. The optical microscope images below each graph show examples of the droplets utilized for these experiments. In the case of small droplets, the laser spot, shown in the central snapshot of panel b, fully overlaps the droplet diameter. Scale bar: $5 \mu \mathrm{m}$.

Further experiments were performed on both PAPT powders and solutions that have been heated in a Raman microscope cell from 25 to $125^{\circ} \mathrm{C}$. In situ Raman analysis was run using a $785 \mathrm{~nm}$ laser source, to avoid any direct laser-induced azo-coupling. Unfortunately, we cannot further extend the temperature range as the spectral background saturates the Raman signal for $\mathrm{T}>130^{\circ} \mathrm{C}$. We note that PAPT is not thermally dimerized within this temperature range. As expected, the spectral background progressively increases as a function of heating, yet its shape is completely different from that of the samples irradiated at $633 \mathrm{~nm}$ and room temperature (Figure S10). Thus, we can conclude that the mere increase of local temperature is not a determining factor for driving the PAPT-to-DMAB transformation. This conclusion agrees with the results reported by Keller and Frontiera on DMAB generation assisted by plasmonic nanoparticles, ${ }^{10}$ yet it contradicts results reported in other works on analogous systems ${ }^{4,11}$ As for the plasmonic counterparts, a possible clue to solve the conundrum is to 
consider the role of atmospheric oxygen. In fact, we observed that PAPT droplets with sizes around 30-40 $\mu \mathrm{m}$, which are unreactive under laser irradiation when freshly deposited, undergo a slow, yet progressive oxidation after being aged in air overnight (Figure S11). Several papers on plasmonic nanoparticle-assisted PAPT-to-DMAB conversion report that no dimerization products are formed when the reaction is carried out in the absence of oxygen, yet Zhao et al. ${ }^{12}$ predicted the possibility to obtain photodimerization without oxygen by means of hot holes and Yan et al. reported the formation of DMAB in $\mathrm{N}_{2}$ atmosphere by adding $\mathrm{AgNO}_{3}$ to $\mathrm{Ag}$ nanoparticles. ${ }^{8}$

The enthalpy of $\mathrm{O}_{2}$ bond is about $498 \mathrm{~kJ} / \mathrm{mol}$, which prevents its direct dissociation under the experimental conditions utilized in this work. In fact, since the energy of each photon emitted at 633 $\mathrm{nm}$ is $3.15 \times 10^{-19} \mathrm{~J}$, the energy flow for a typical full power irradiation microRaman experiment is limited to $1.6 \mathrm{~mJ} / \mu \mathrm{m}^{2} \mathrm{~s}$. Moreover, the inertness of PAPT irradiated in air at $785 \mathrm{~nm}$ and in-situ thermal heating control experiments points out to a wavelength-dependent photochemical activation of oxygen. This hypothesis was assessed through a series of confocal Raman microspectroscopy experiments carried out on PAPT powders. The PAPT crystals were analyzed with and without atmospheric oxygen. Samples irradiated in air show a progressive increase of the spectral background and the appearance of DMAB weak Raman modes. The optical microscope inspection of the crystals after irradiation reveals the formation of circular regions that are commensurate to the footprint of the laser beamsize, indicating the partial degradation of the sample surface (Figure 3a).

On the other hand, when the same experiment is carried out by focusing the laser beam not on the surface but deep inside the crystal, the Raman spectrum does not show any modification, both in terms of background and appearance of new modes (Figure 3b). This means that laser irradiation per $s e$ is not sufficient to trigger dimerization and confirms the key role of oxygen in the process. However, unlike all previous hypotheses reported in literature, these results suggest that oxygen can be involved in dimerization only if activated by laser irradiation. In this regard, we note that irradiation at $633 \mathrm{~nm}(1.96 \mathrm{eV})$ can directly promote the excitation of molecular oxygen to singlet oxygen states $\left(\mathrm{O}_{2} \mathrm{~b}^{1} \sum_{\mathrm{g}}{ }^{+}\right.$and $\left.\mathrm{O}_{2} \mathrm{a}^{1} \Delta_{\mathrm{g}}\right)$ with both monomol and dimol transitions. ${ }^{13}$ 

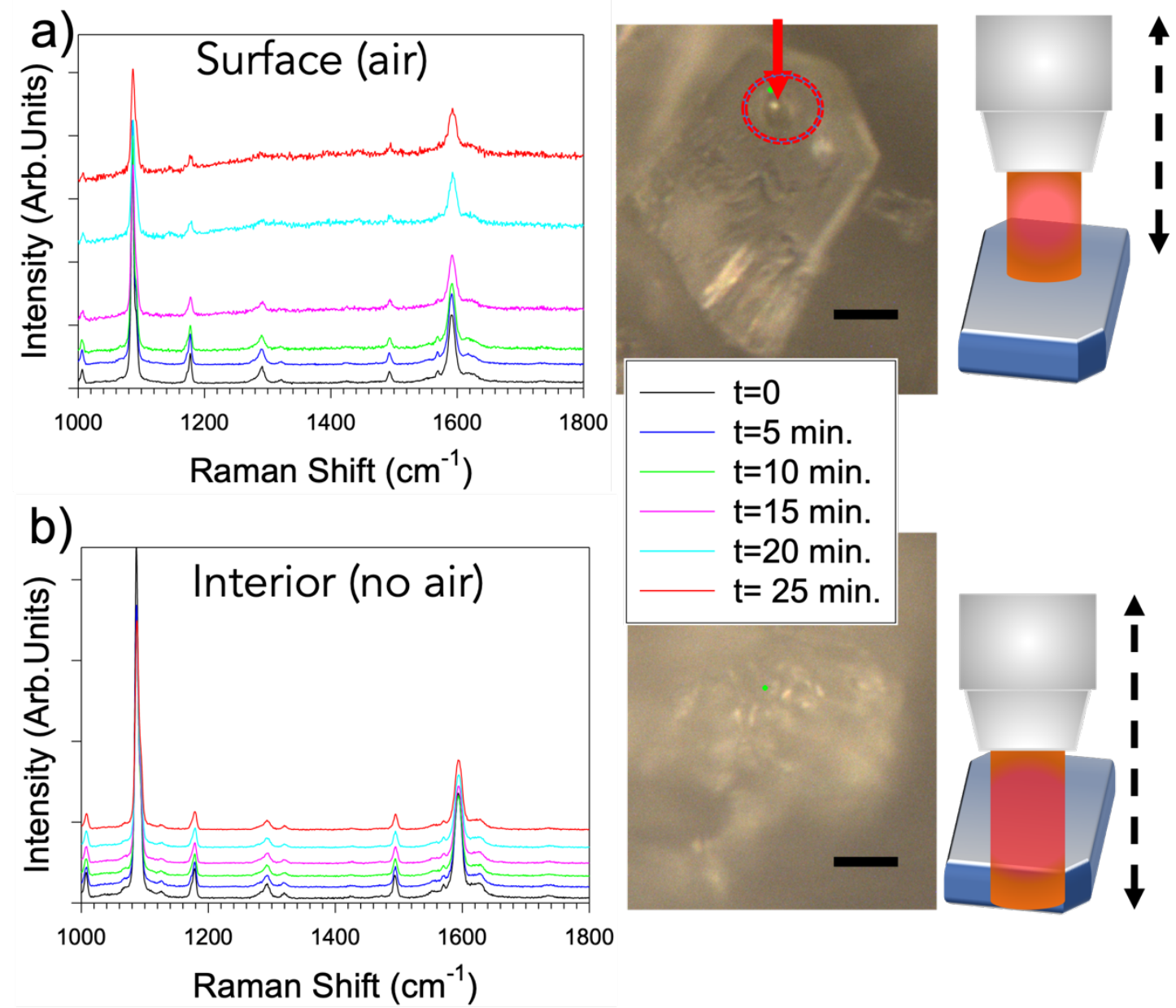

$=5$ min. $\mathrm{t}=10 \mathrm{~min}$. $\mathrm{t}=15 \mathrm{~min}$. $\mathrm{t}=20 \mathrm{~min}$. $\mathrm{t}=25 \mathrm{~min}$.

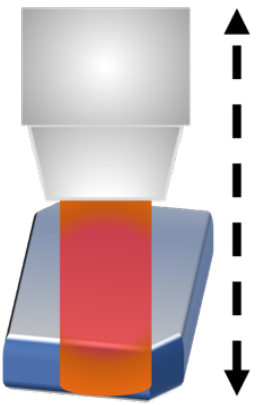

Figure 3. Temporal evolution of the Raman spectra of PAPT powders irradiated at $633 \mathrm{~nm}, 1.6$ $\mathrm{mW} / \mu \mathrm{m}^{2}$ on a) surface and b) inner (bulk) regions of the crystals, obtained by confocal microscopy acquisition at different penetration depth (see the scheme on the right). The spectra acquired from the inner regions of the samples are analogous to those acquired in nitrogen-saturated atmosphere. The corresponding optical microscope images are shown (scale bar: $5 \mu \mathrm{m}$ ). In the case of surface focusing (Panel a), the formation of the laser footprint is indicated by the red circle. No morphological modification was observed either for interior regions or oxygen-free surfaces (Panel b).

In monomol transition a single ground-state $\mathrm{O}_{2}$ molecule $\left({ }^{3} \mathrm{O}_{2}\right)$ can be photoexcited to either $\mathrm{O}_{2} \mathrm{~b}^{1} \Sigma_{\mathrm{g}}{ }^{+}$ (highest energy) or $\mathrm{O}_{2} \mathrm{a}^{1} \Delta_{\mathrm{g}}$ (lowest energy) states. If the photon energy is above $1.62 \mathrm{eV}$ the $\mathrm{O}_{2}$ $\mathrm{b}^{1} \Sigma_{\mathrm{g}}{ }^{+}$level can be reached, which undergoes rapid de-excitation to the $\mathrm{O}_{2} \mathrm{a}^{1} \Delta_{\mathrm{g}}$ state. In parallel, dimol transitions can be stimulated at $633 \mathrm{~nm}$ as well. In this type of transitions, whose absorption coefficient is very low, one photon is absorbed by two ground state oxygen molecules linked in a transient complex $\left[{ }^{3} \mathrm{O}_{2}-{ }^{3} \mathrm{O}_{2}\right]$, producing two singlet oxygen species. In any case, singlet oxygen species are strongly oxidating agents that can easily promote the generation of oxygen radical species. 
The latter can trigger and sustain the oxidation of PAPT into carbonaceous species, which passes through the formation of DMAB (Figure 4). This hypothesis could explain the observation of different reaction kinetics in differently sized PAPT droplets. As schematically illustrated in Figure 4, small droplets are probably more reactive than their bigger counterparts because they are commensurate to the laser beam size, which ranges between 1.5 and $2.12 \mu \mathrm{m}$. This allows for a direct excitation of almost all $\mathrm{O}_{2}$ molecules physisorbed all around the droplet surface. As the laser excitation is continuous wave-type, photons are constantly supplied, and local power becomes a critical parameter to control the extent of photo-oxidation over time.
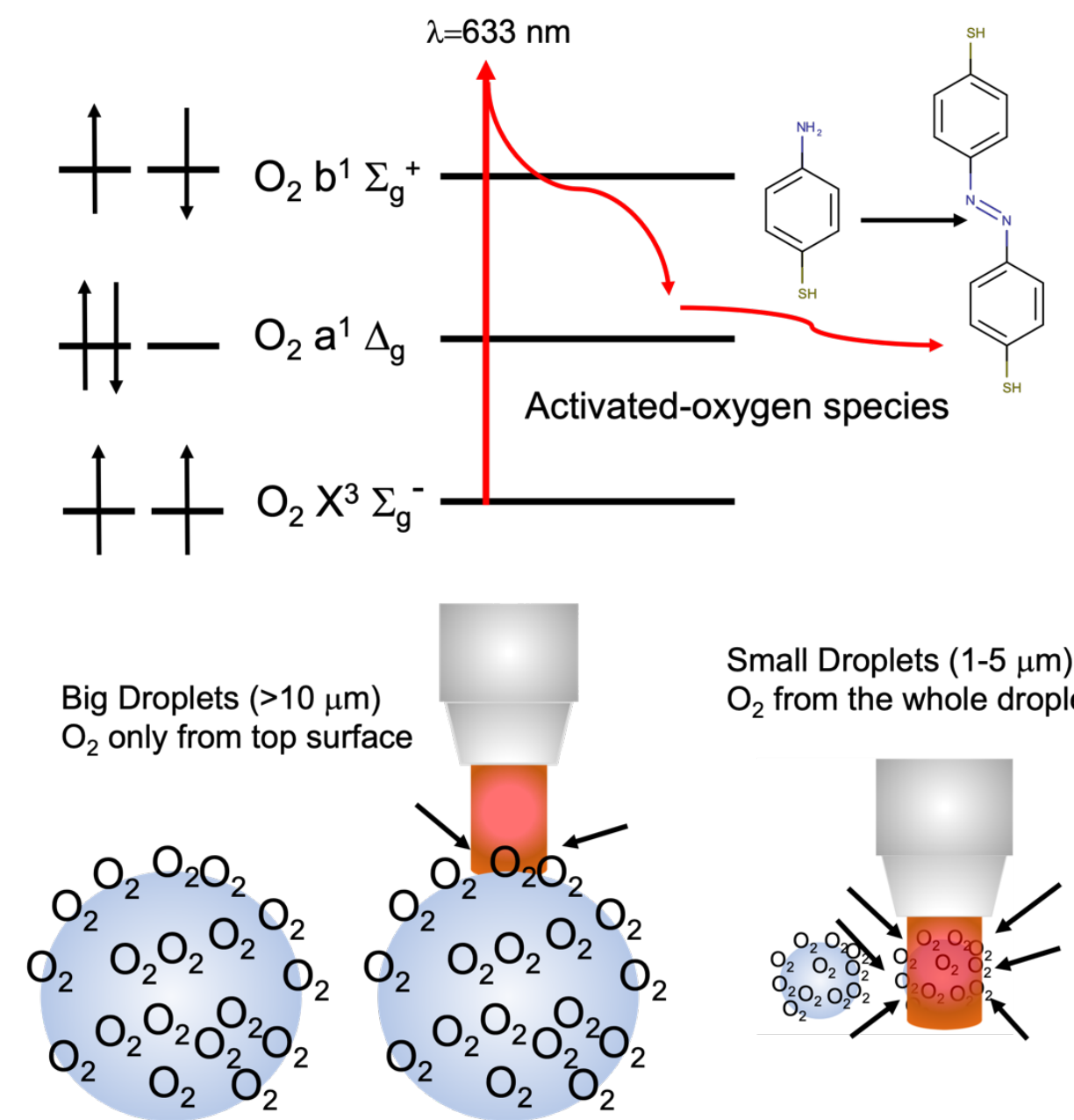

Figure 4. Scheme of the model of plasmon-free photo-oxidation of PAPT. The He-Ne laser $(\lambda=633$ $\mathrm{nm}, 1.96 \mathrm{eV}$ ) can directly excite the transition of $\mathrm{O}_{2}$ from triplet to a singlet state $\mathrm{O}_{2} \mathrm{~b}^{1} \sum_{\mathrm{g}^{+}}$, which eventually decays to a $\mathrm{O}_{2} \mathrm{a}^{1} \Delta \mathrm{g}$ state. Both types of singlet states can concur in promoting the photooxidation of PAPT. In the case of droplets significantly bigger than the laser beam size only $\mathrm{O}_{2}$ molecules adsorbed on the top surface can be excited, whereas irradiation of small droplets involves almost the whole sphere surface, which is about 4 times larger, making photo-oxidation more efficient. 
On the other hand, in the case of bigger droplets this excitation is limited to the portion of surface defined by the beam size. In this case the amount of $\mathrm{O}_{2}$ molecules is not sufficient to activate and extend oxidation above the detection limit. On the other hand, when aged in air for several hours, bigger droplets are allowed to adsorb more oxygen, thus the probability of generating oxidizing species by photoactivation is increased. Similarly, the Raman experiments on $\mathrm{TiO}_{2}$ nanoparticles, microspheres and thin films can be interpreted according to this model. The higher the sample surface area, the higher $\mathrm{O}_{2}$ adsorption and the faster the photo-oxidation.

Thus, the fact that no dimerization was observed in some previous reports ${ }^{7}$ upon irradiation at 633 $\mathrm{nm}$, even in the presence of plasmonic nanoparticles, may depend on the fact that in aqueous solution the concentration of dissolved oxygen is below $0.27 \mathrm{mM}$, which makes the generation of oxygen photo-excited species quite inefficient. Moreover, irradiation time was too short $(1 \mathrm{~s})$, to generate a sufficient amount of photoactivated oxygen species for triggering the reaction. Other works reported indeed the occurrence of photodimerization at $633 \mathrm{~nm}$ in air and the role of oxygen species photoactivated with the assistance of plasmonic nanoparticles has been demonstrated.9.14-16 What is new in the present work is the discovery that PAPT can be transformed by photoactivated oxygen even in the absence of any plasmonic mediators. This means that hot carriers may be helpful to assist the generation of reactive oxygen species ( ${ }^{1} \mathrm{O}_{2}$ and derivatives) but not necessary to drive a process that has been normally considered the paradigm of plasmon-driven reactions. The beneficial role of nanoparticles could be not only limited to boost the generation of reactive oxygen species, but it could be associated to molecule binding and high surface areas. Plasmonic nanoparticles provide an excellent surface to bind thiol groups, which constrains the PAPT molecules to form self-assembled monolayers. Although often neglected, this regular proximity could play a key role in promoting dimerization. On the other hand, the formation of disulfide bridges and hydrogen bonds hamper photodimerization, thus films made from highly concentrated solutions or powdered samples are relatively inert. In parallel, the high reactivity of $\mathrm{TiO}_{2}$ nanoparticles demonstrates the importance of maximizing oxygen adsorption. 
Overall, this work widens the horizons of light-driven surface reactions, showing that oxygen photoactivation, which is involved in a variety of chemical and biochemical processes, can be achieved and controlled by morphology factors, without need of any charge-transfer mediators, such as plasmonic nanoparticles.

In perspective, the study of laser-induced production of oxygen photoactivated species, so far limited to photobiology and phototherapy, may open new frontiers for surface photochemistry and provide new clues to interpret SERS spectra characterized by low temporal stability and ill-defined "chemical enhancement". ${ }^{17}$

\section{Acknowledgements}

Thanks to Dr. Marco Gandolfi, Dr. Andrea Tognazzi and Prof. Costantino De Angelis for valuable discussions.

\section{Supporting Information}

Experimental details and control experiments are reported in SI.

\section{References}

(1) Osawa, M.; Matsuda, N.; Yoshii, K; Uchida I. Charge Transfer Resonance Raman Process in Surface-Enhanced Raman Scattering J.Phys. Chem. 1994, 98, 12702-12707

(2) Huang, Y.-F.; Zhu, H-P.; Liu, G-K.; Wu, D-Y; Ren B.; Tian, Z-Q. When the Signal is not from the Original Molecule to be Detected. Chemical Transformation of ParaAminothiophenol on Ag during the SERS Measurement. J. Am. Chem. Soc. 2010, 132, 92449246. 
(3) Devasenathipathy, R.; Rani, K. K.; Liu, J.; Wu, D-Y; Tian Z-Q. Plasmon Mediated Photoelectrochemical Transformations: The Example of Para-Aminothiophenol, Electrochim. Acta 2021, 367, 137485

(4) Sarhan R. M.; Koopman, W.; Schuetz, R.; Schimd, T.; Liebig, F.; Koetz, J.; Bargheer, M., The Importance of Plasmonic Heating for the Plasmon-Driven Photodimerization of 4Nitrophenol, Sci.Rep. 2019 9, 3060, DOI: 10.1038/s41598-019-38627-2

(5) Y-F. Huang, M. Zhang, L-B. Zhao, J-M. Feng, D-Y Wu, B. Ren, Tian Z-Q. Activation of Oxygen on Gold and Silver Nanoparticles Assisted by Surface Plasmon Resonances. Angew. Chem. Int. Ed. 2014, 53, 2353-2357.

(6) Zhang, Z.; Kneipp, J. Ligand-Supported Hot Electron Harvesting: Revisiting the pHResponsive Surface-Enhanced Raman Scattering Spectrum of p-Aminothiophenol. J. Phys. Chem. Lett. 2021, 12, 1542-1547.

(7) Zhang, Z.; Kinzel. D.; Deckert W. Photo-Induced or Plasmon-Induced Reaction: Investigation of the Light-Induced Azo-Coupling of Amino Groups. J. Phys. Chem. C 2016, 120, 20978-20983.

(8) Yan, X.; Wang, L.; Tan, X.; Tian, B.; Zhang, J. Surface-Enhanced Raman Spectroscopy Assisted by Radical Captures Sci Rep. 2016, DOI: 10.1038/srep30193;

(9) Xu, P.; Kang, L.; Mack, N. H.; Schanze, K. S.; Han, X.; Wang, H-L. Mechanistic understanding of surface plasmon assisted catalysis on a single particle: cyclic redox of 4aminothiphenol, Sci Rep 2013, 3: 2997, DOI: 10.1038/srep02997

(10) Keller, E. L.; Frontiera, R. R. Ultrafast Nanoscale Raman Thermometry Proves Heating Is Not a Primary Mechanism for Plasmon-Driven Photocatalysis. ACS Nano 2018, 12, 6, 58485855.

(11) Golubev, A. A.; Khlebtsov, B. N.; Rodriguez, R. D.; Chen, Y.; Zhan, D. R. Plasmonic Heating Plays a Dominant Role in the Plasmon-Induced Photocatalytic Reduction of 4Nitrobenzenthiol. J. Phys. Chem. C 2018, 122, 5657-5663. 
(12) Zhao, L-B.; Zhang, M.; Huang, Y-F.; Williams, C. T.; Wu, D. Y.; Ren, B.; Tian, Z-Q. Theoretical Study of Plasmon-Enhanced Surface Catalytic Coupling Reactions of Aromatic Amines and Nitro Compounds. J. Phys. Chem. Lett. 2014, 5, 1259-1266.

(13) Blazquez-Castro, A. Direct ${ }^{1} \mathrm{O}_{2}$ optical excitation: A Tool for Redox Biology. Redox Biol. 2017, 13, 39-59.

(14) Huang, Y-F.; Zhang, M.; Zhao, L-B.; Feng, J-M.; Wu, D-Y.; Ren, B.; Tian, Z-Q. Activation of Oxygen on Gold and Silver Nanoparticles Assisted by Surface Plasmon Resonances, Angew. Chem. Int. Ed. 2014, 53, 2353-2357.

(15) Zhang, Y.; Aslan, K.; Previte, M. J. R.; Geddes, C. D. Plasmonic Engineering of Singlet Oxygen Generation. Proc. Natl. Acad.Sci. 2008, 105, 1798-1802.

(16) Salmistraro. M.; Schwartzberg, A.; Bao, W.; Depero, L. E.; Weber-Bargioni, A.; Cabrini, S.; Alessandri, I. Triggering and Monitoring Plasmon-Enhanced Reactions by Optical Nanoantennas Coupled to Photocatalytic Beads, Small 2013, 9, 3301-3307.

(17) Moskovits, M. Surface-Enhanced Spectroscopy. Rev. Mod. Phys. 1985, 57, 783-826.

(18) Alessandri, I. Enhancing Raman Scattering without Plasmons: Unprecedented Sensitivity Achieved by $\mathrm{TiO}_{2}$ Shell-Based Resonators J. Am. Chem. Soc. 2013, 135, 5541-5544. 


\title{
SUPPORTING INFORMATION
}

\section{4-Aminothiophenol Photodimerization Without}

\section{Plasmons}

\author{
Ivano Alessandri ${ }^{1,2,3 *}$
}

${ }^{1}$ Sustainable Chemistry and Materials Laboratory, Department of Information Engineering, University of Brescia (Italy)

${ }^{2}$ INSTM, Consorzio Nazionale per la Scienza e Tecnologia dei Materiali, RU Brescia, via Branze 38, 25123 Brescia (Italy)

${ }^{3}$ INO-CNR, RU Brescia, via Branze 43, 25123 Brescia (Italy)

e-mail: ivano.alessandri@unibs.it

\section{Table of Contents}

S0. Experimental Details

S1-S6 Control experiments on $\mathrm{TiO}_{2}$ supports

S7-S11 Control experiments on PAPT droplets 


\section{So. Experimental Details}

\section{Chemicals}

4-aminothiophenol powder (purity $>97 \%$ ) and ethanol $(99.8 \%$ ) were purchased by Merck-SigmaAldrich and utilized without any further purification.

The technical specifications of commercial P25- $\mathrm{TiO}_{2}$ Aeroxide ${ }^{\circledR}$ nanopowders (Evonik) can be found in the online version of material datasheet. ${ }^{1}$ The $\mathrm{SiO}_{2} / \mathrm{TiO}_{2}$ core/shell microspheres (T-rex) were fabricated by following the experimental protocol reported in ref. 2. ${ }^{2}$ The planar thin films were achieved with the same procedure, without using silica spheres.

\section{Confocal microRaman experiments}

The confocal microRaman experiments were carried out by a high-resolution Raman microscope (Labram HR-800, Horiba/Jobin-Yvon), equipped a Syncerity CCD detector and an Olympus B-41 microscope stage, with 4 optical objectives (10X, 50X, 50X Long Working Distance, LWD and 100X). The optical images were acquired directly from the Raman microscope stage. The Raman spectra were acquired with a 50X LWD objective (Numerical Aperture, N. A. 0.50). The in-situ, temperature Raman measurements were carried out with a Linkam HFS-91 thermal stage, using the same optical objective. This stage was also utilized for the measurements in the absence of oxygen. The anoxic conditions were obtained by saturating the chamber with pure nitrogen. Confocal analyses to differentiate surface and interior of the PAPT crystals were carried out by progressively changing the focal plane through the crystals under analysis.

At least 10 different regions were analyzed for each sample tested in Raman experiments.

\section{References:}

1) $\mathrm{P} 25-\mathrm{TiO}_{2}$ Aeroxide ${ }^{\circledR}$ datasheet available at products-re.evonik.com

2) Alessandri, I. Enhancing Raman Scattering without Plasmons: Unprecedented Sensitivity Achieved by $\mathrm{TiO}_{2}$ Shell-Based Resonators J. Am. Chem. Soc. 2013, 135, 5541-5544 


\section{S1. Control Experiment 1}

Laser irradiation: $633 \mathrm{~nm}, 1.6 \mathrm{~mW} / \mu \mathrm{m}^{2}$
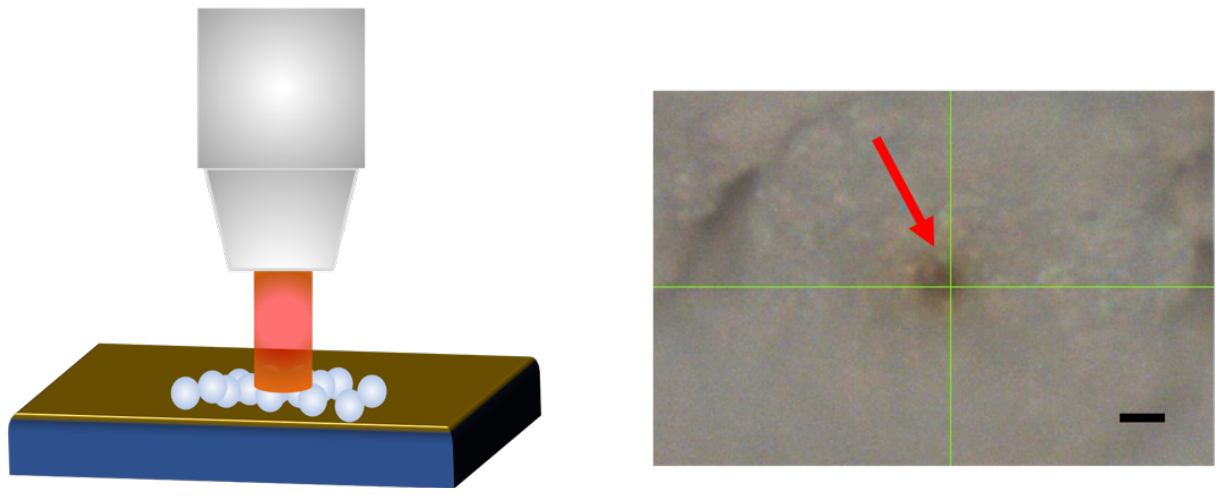

Figure S1. Optical microscope image showing the effects of He-Ne $(\lambda=633 \mathrm{~nm})$ laser irradiation at $1.6 \mathrm{~mW} / \mu \mathrm{m}^{2}$ on P25 nanopowders previously soaked in a $10^{-4} \mathrm{M}$ PAPT ethanol solution. The dark dot indicated by the red arrow shows the laser footprint. Scale bar: $5 \mu \mathrm{m}$.

\section{S2. Control Experiment 2}

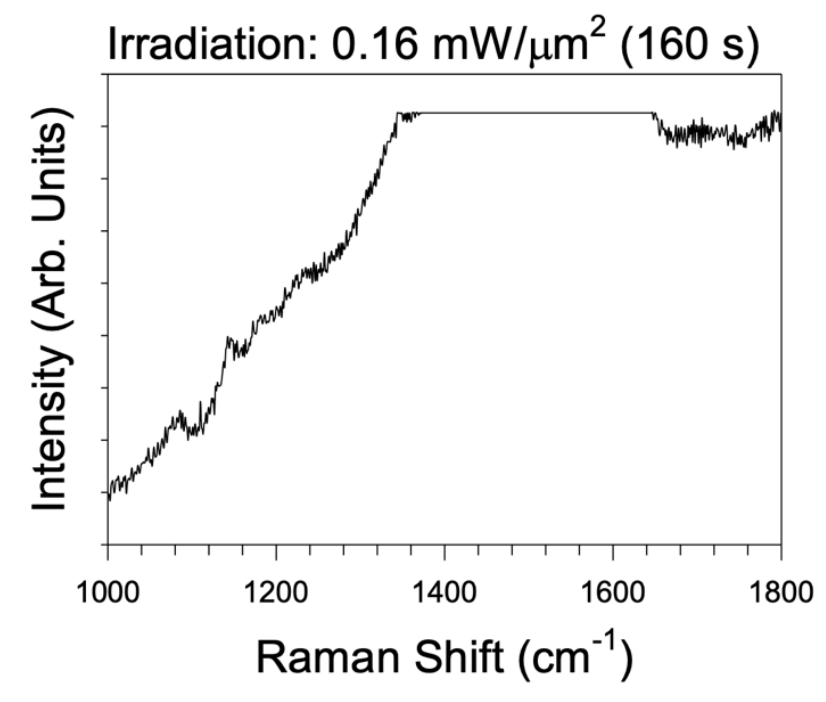

Figure S2. Raman spectrum of $\mathrm{P} 25 \mathrm{TiO}_{2}$ nanopowders previously soaked in a $10^{-4} \mathrm{M}$ PAPT ethanol solution, irradiated at $633 \mathrm{~nm}, 0.16 \mathrm{~mW} / \mu^{2}$ for $160 \mathrm{~s}$, showing the saturation of the signal in the $1350-1800 \mathrm{~cm}^{-1}$ region. 


\section{S3. Control Experiment 3}

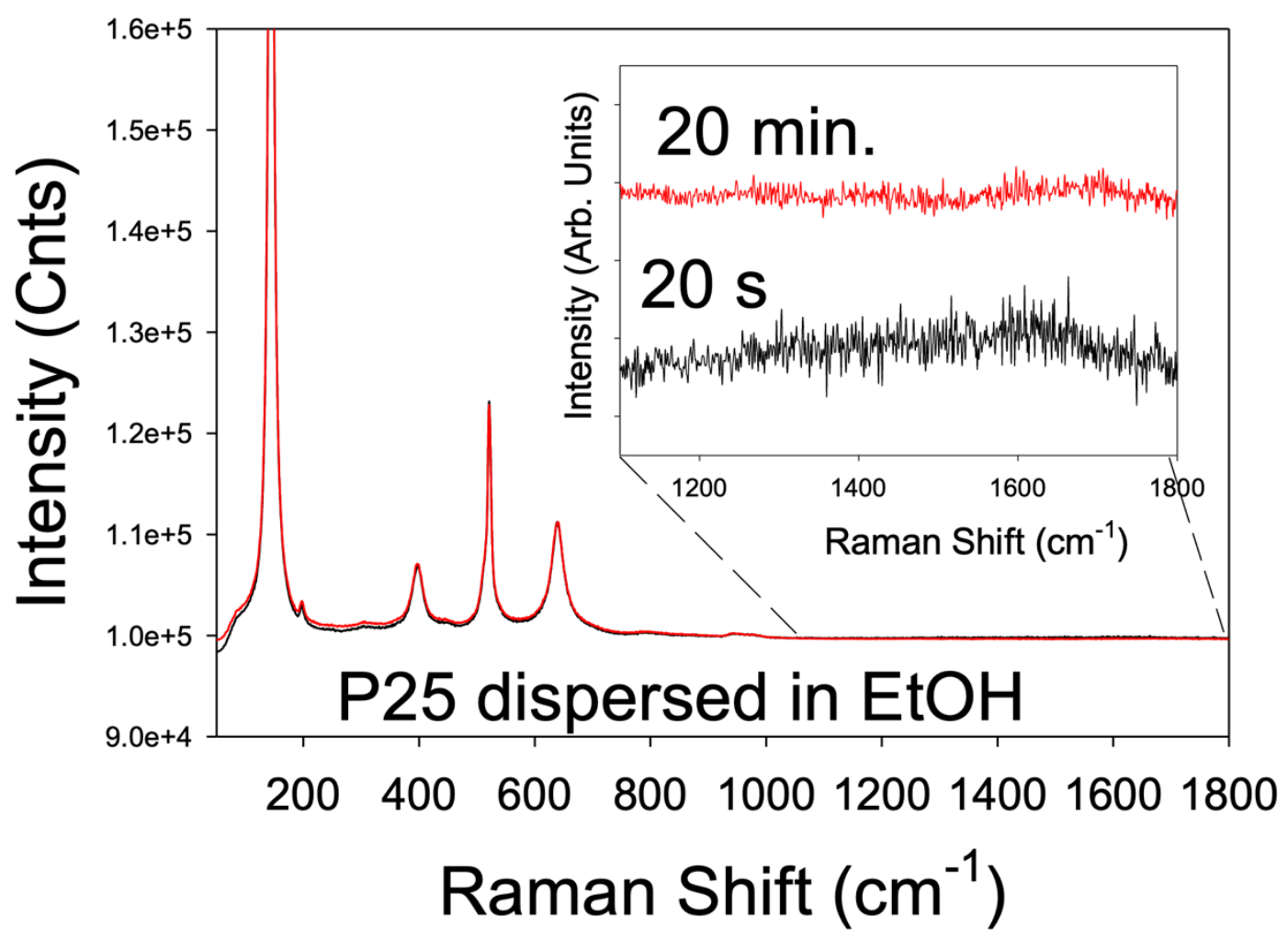

Figure S3. Raman spectrum of P25 soaked in ethanol in the absence of PAPT and irradiated under the same conditions $\left(633 \mathrm{~nm}, 0.16 \mathrm{~mW} / \mu \mathrm{m}^{2}\right)$ of experiment shown in Figure 1 (main text). The Raman spectra have been stacked for clarity. The inset shows a zoomed view of the $1000-1800 \mathrm{~cm}^{-1}$ region. 


\section{S4. Control Experiment 4}

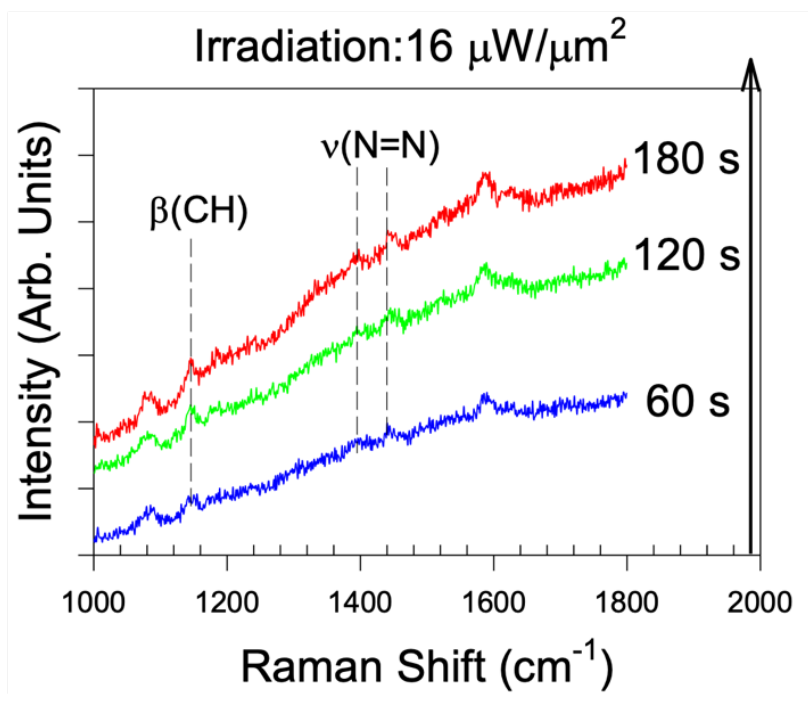

Figure S4. Raman spectra of $\mathrm{P} 25 \mathrm{TiO}_{2}$ nanopowders previously soaked in a $10^{-4} \mathrm{M}$ PAPT ethanol solution, irradiated at $633 \mathrm{~nm}, 16 \mu \mathrm{W} / \mu \mathrm{m}^{2}$ ). The Raman spectra have been stacked for clarity. The inset shows a zoomed view of the $1000-1800 \mathrm{~cm}^{-1}$ region.

\section{S5. Control Experiment 5}

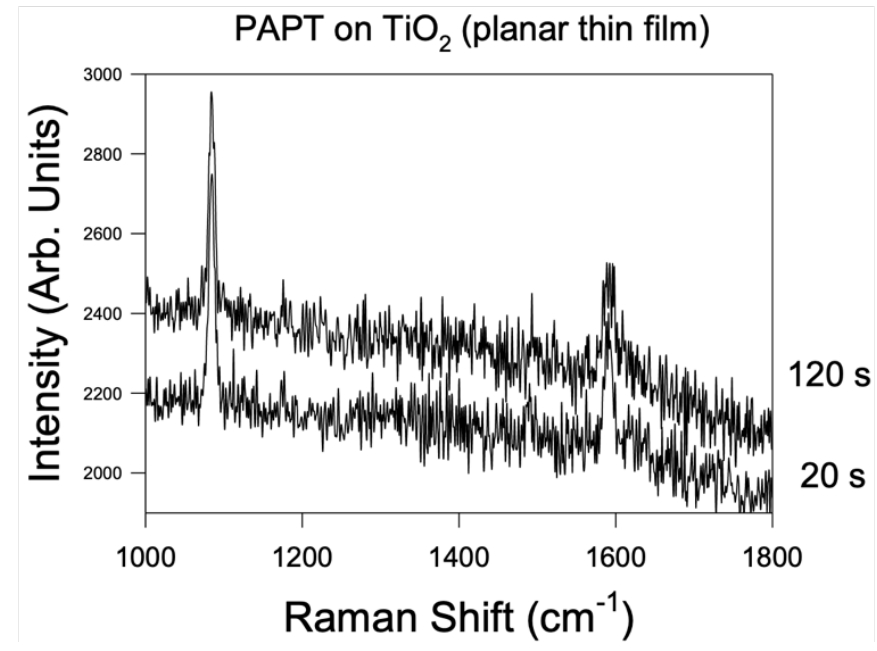

Figure S5. Raman spectra of anatase $\mathrm{TiO}_{2}$ planar thin films previously soaked in a PAPT $10^{-4} \mathrm{M}$ ethanol solution irradiated at $633 \mathrm{~nm}, 0.16 \mathrm{~mW} / \mu \mathrm{m}^{2}$ ). The Raman spectra have been stacked for clarity. 


\section{S6. Control Experiment 6}

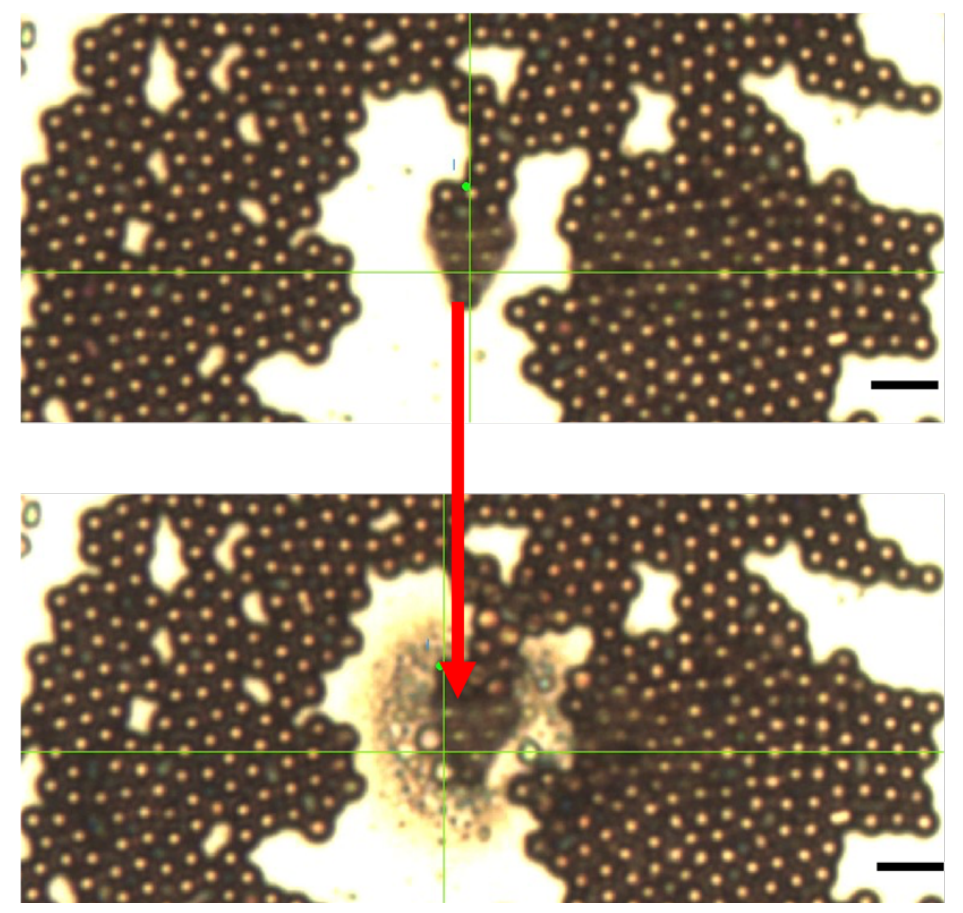

Figure S6. Optical microscope images showing the effects of $633 \mathrm{~nm}$ laser irradiation $\left(1.6 \mathrm{~mW} / \mu \mathrm{m}^{2}\right)$ on $\mathrm{SiO}_{2} / \mathrm{TiO}_{2}$ core/shell microspheres (T-rex beads). Scale bars: $5 \mu \mathrm{m}$.

\section{S7. Control Experiment 7}
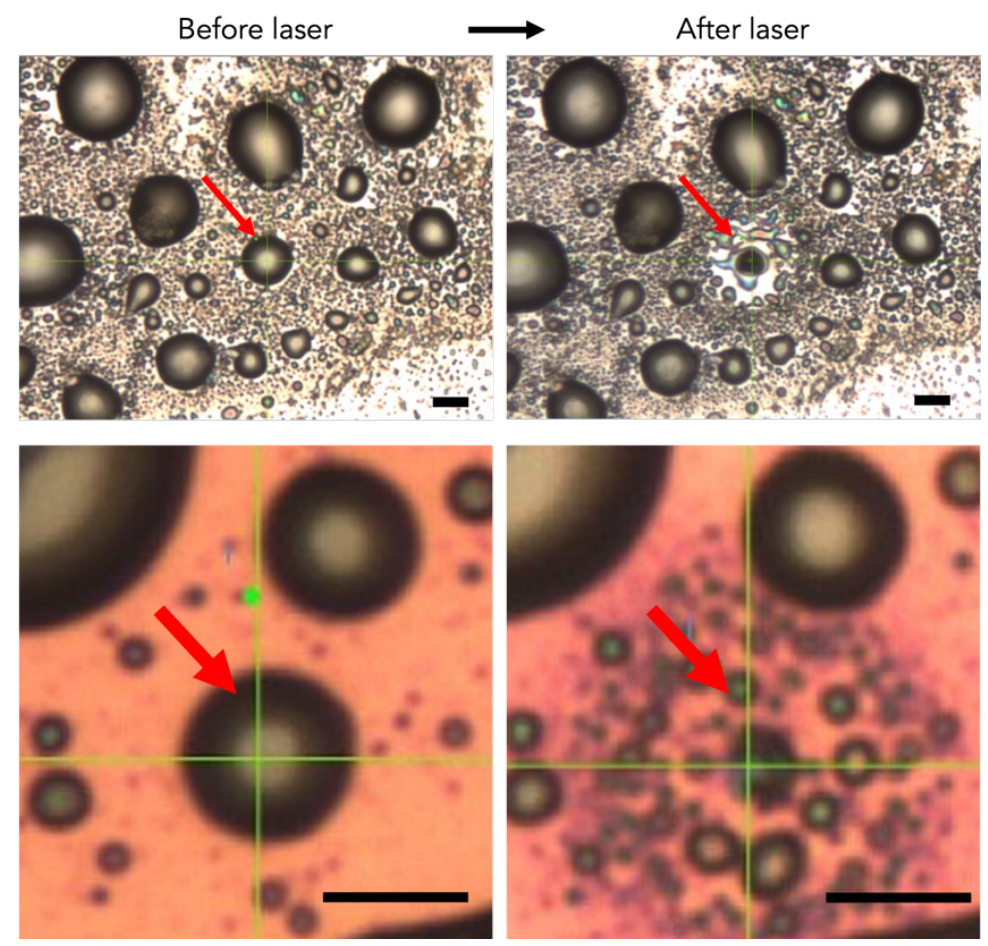

Figure S7. Optical microscope images showing examples of the effects of $633 \mathrm{~nm}$ laser irradiation $\left(1.6 \mathrm{~mW} / \mu \mathrm{m}^{2}\right)$ on PAPT small droplets. Scale bars: $5 \mu \mathrm{m}$. 


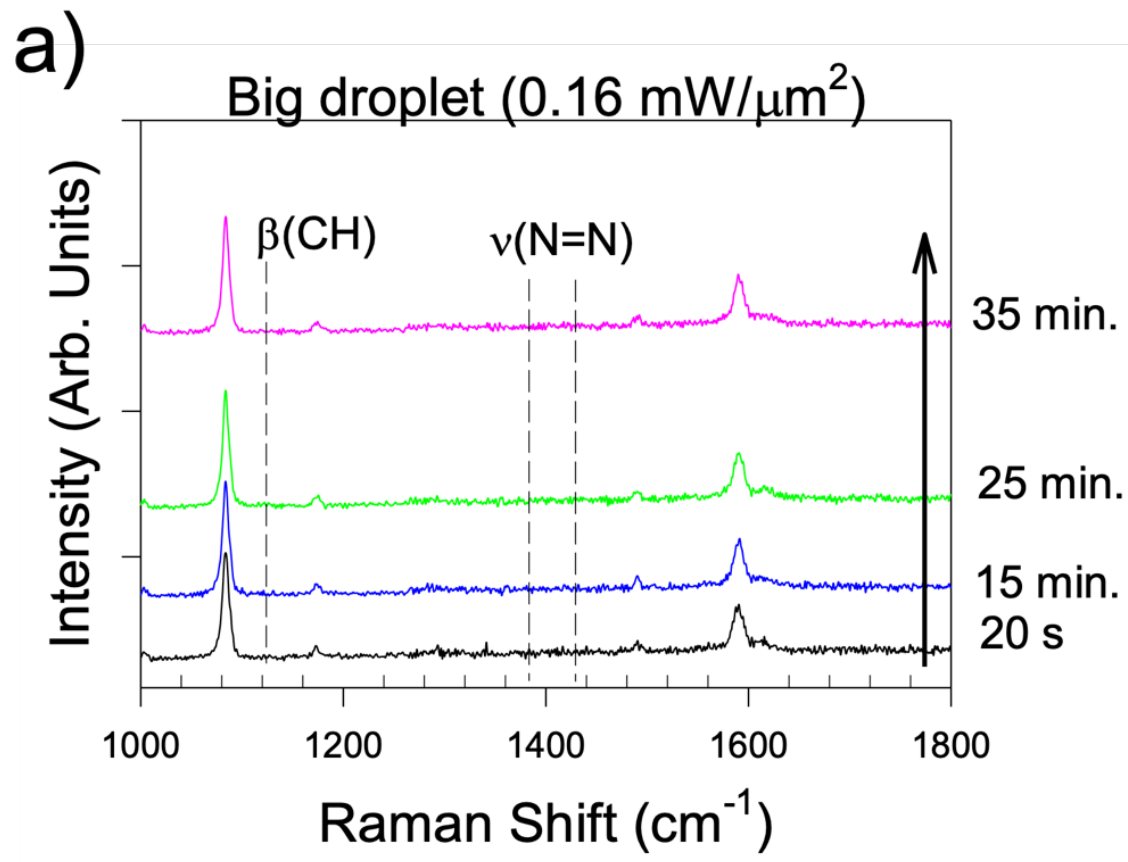

b) Small droplet $\left(0.16 \mathrm{~mW} / \mu \mathrm{m}^{2}\right)$

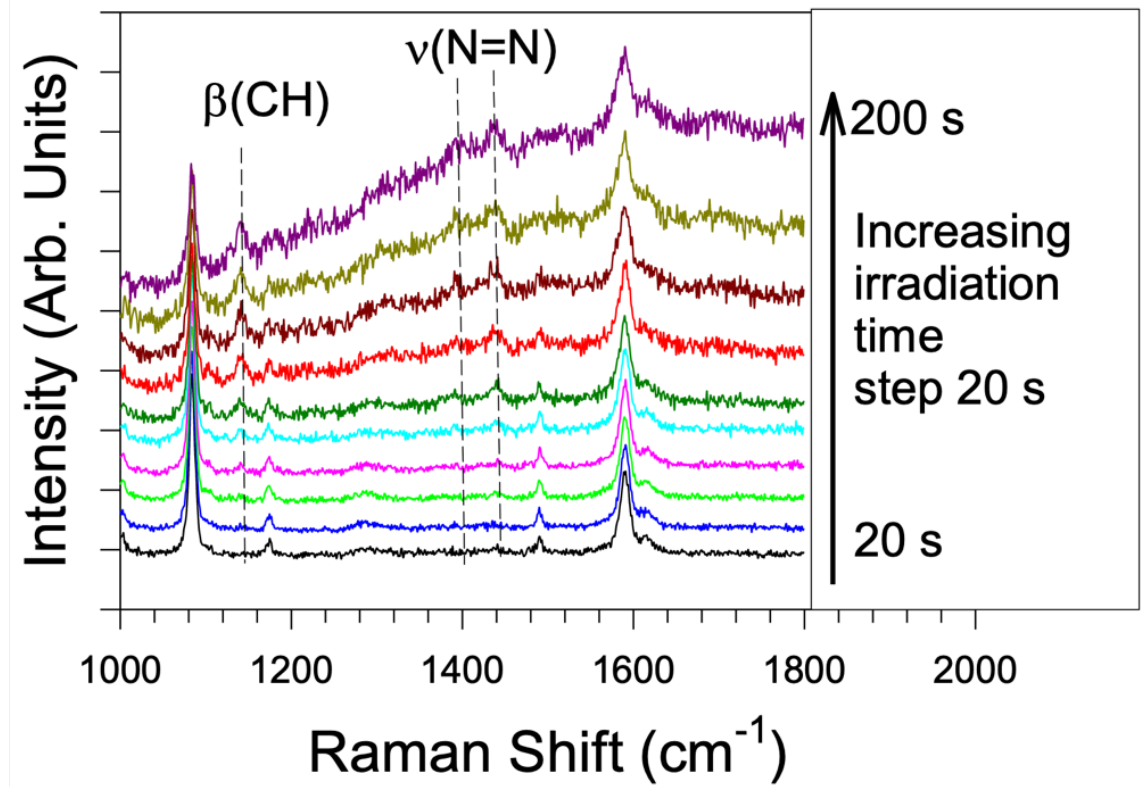

Figure S8. Temporal evolution of the PAPT (and DMAB) Raman spectra for a) big (i.e. $>30 \mu \mathrm{m}$ ) and small $($ i.e. $<5 \mu \mathrm{m})$ droplets, irradiated at $0.16 \mathrm{~mW} / \mu \mathrm{m}^{2}$. 


\section{Small droplet irradiated at $785 \mathrm{~nm}$}

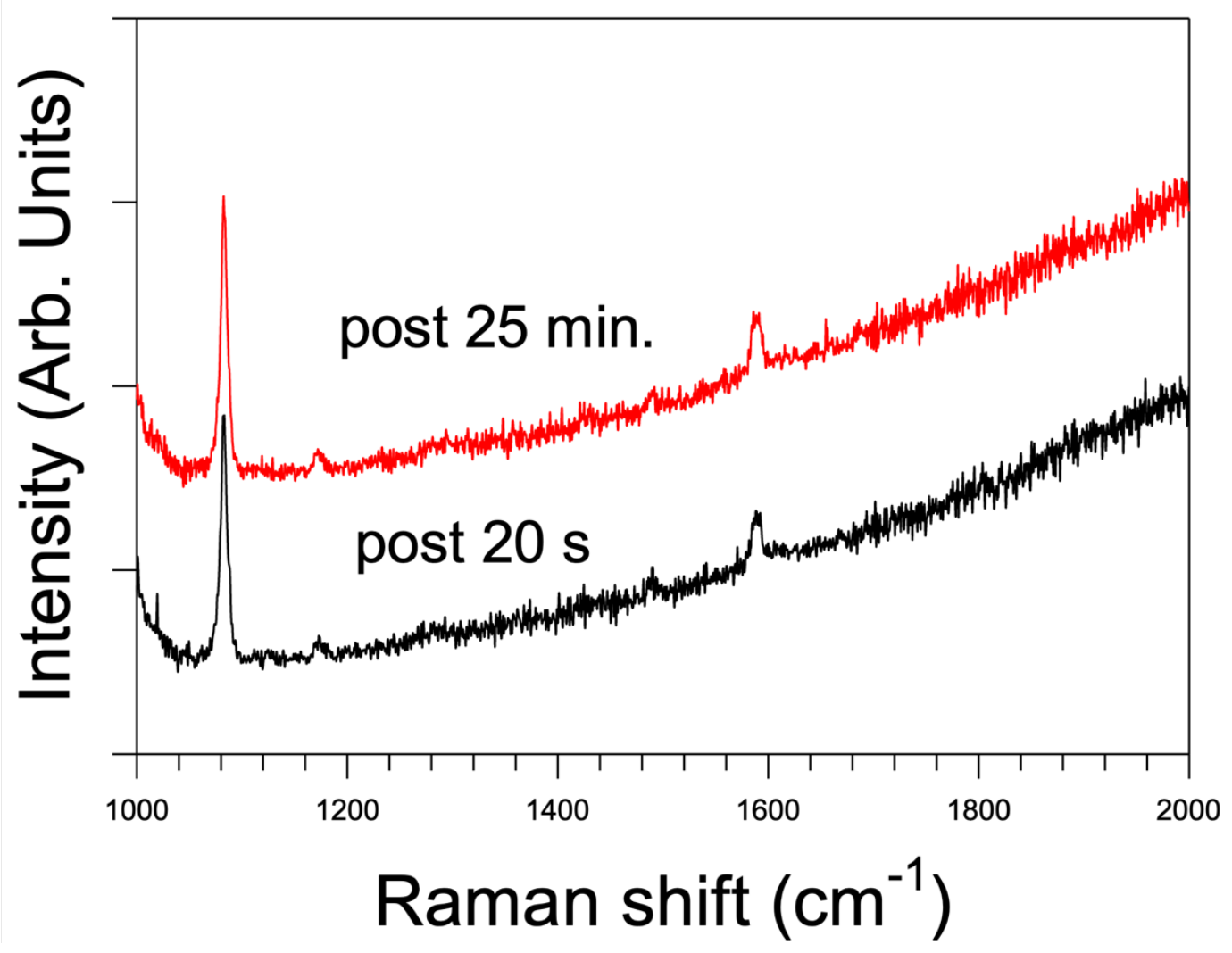

Figure S9. Raman spectra of PAPT small $(<5 \mu \mathrm{m})$ droplets irradiated at $785 \mathrm{~nm}\left(260 \mathrm{~mW} / \mu \mathrm{m}^{2}\right)$ at different exposure time. The spectra were stacked for clarity. 


\section{In situ thermal heating at $785 \mathrm{~nm}$}

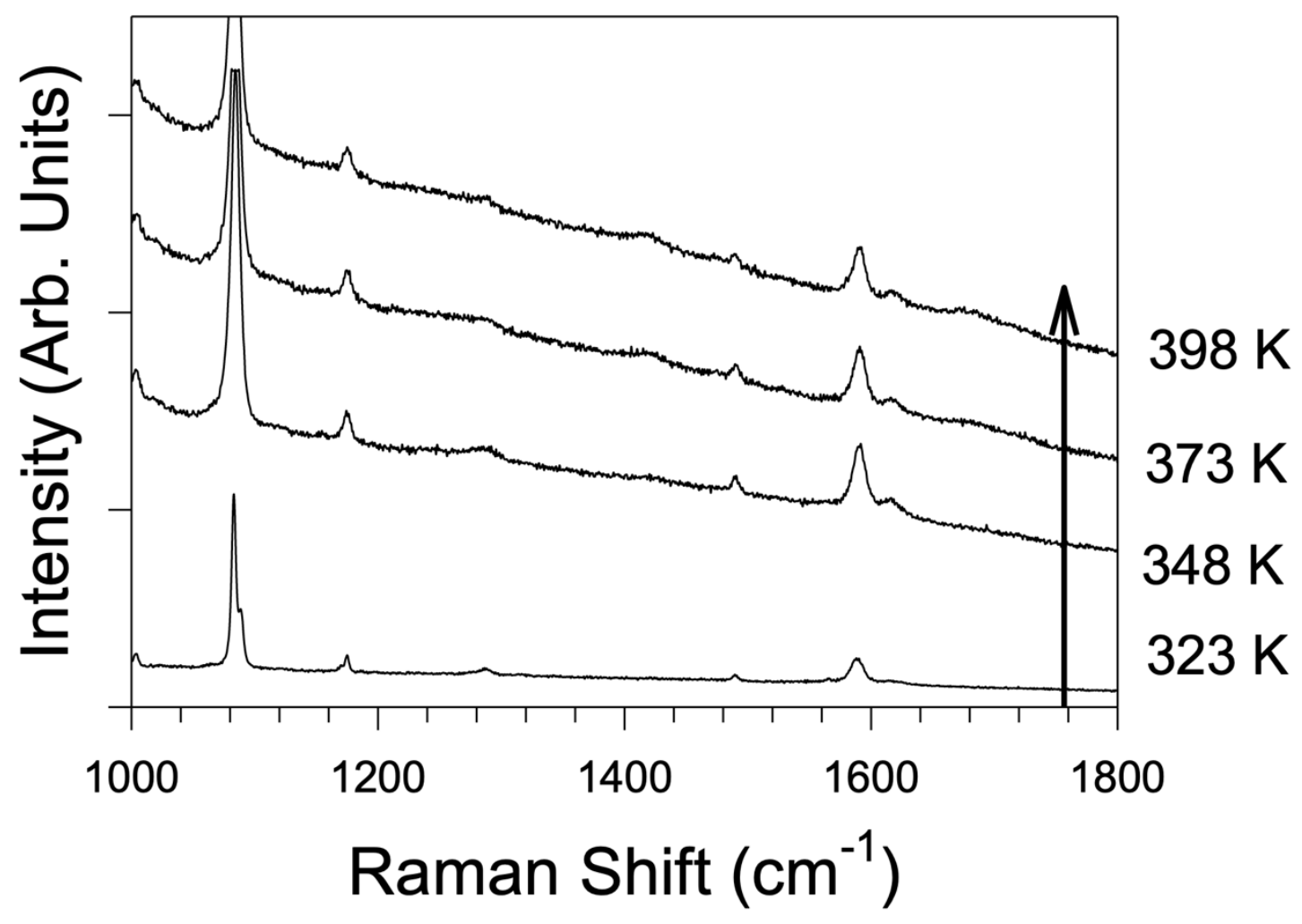

Figure S10. In-situ thermal heating of PAPT powders irradiated at $785 \mathrm{~nm}$ in a Linkam cell. The acquisition time for each scan was $10 \mathrm{~s}$. 
Big droplet aged in air overnight $\left(1.6 \mathrm{~mW} / \mu \mathrm{m}^{2}\right)$

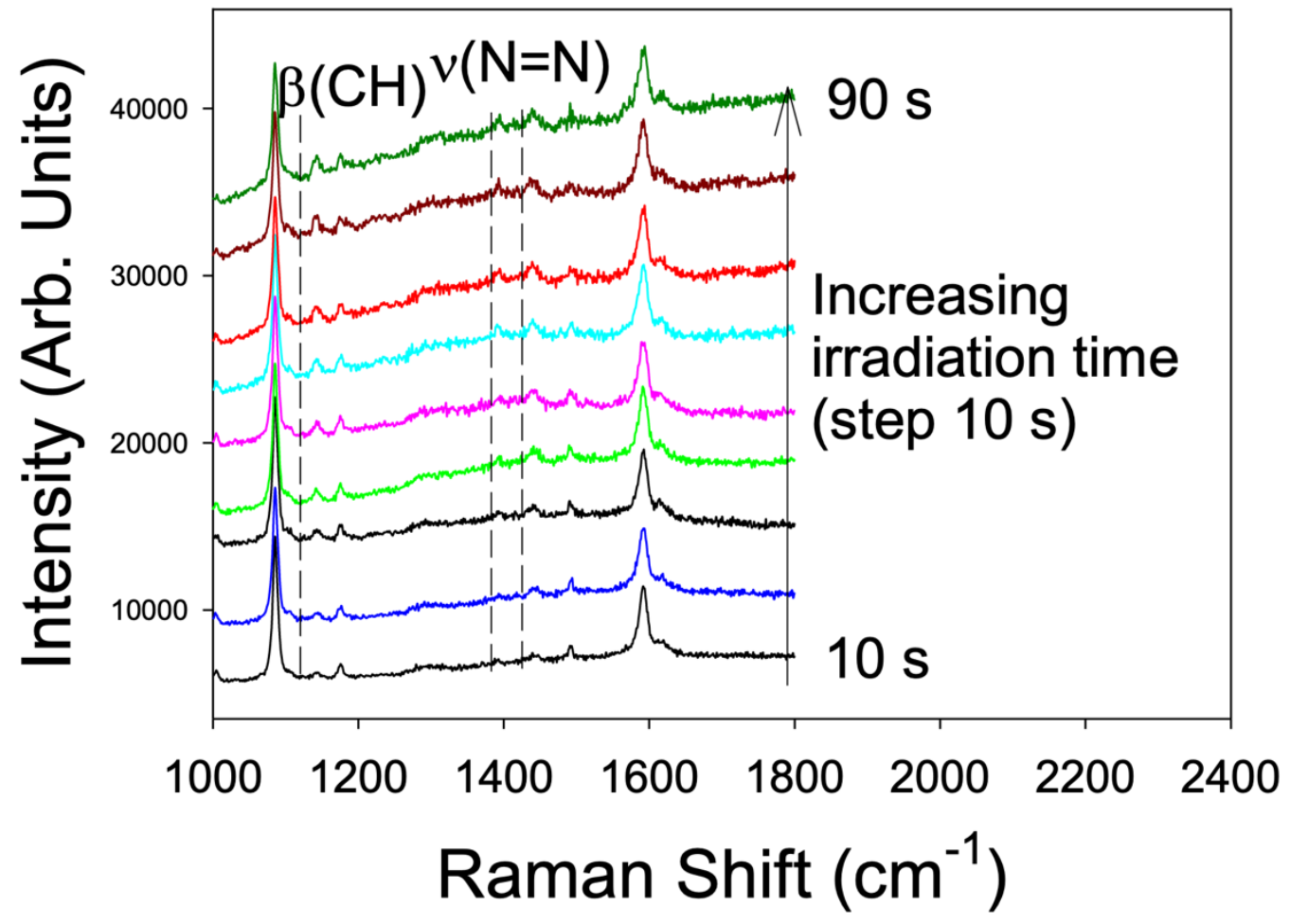

Figure S11. Temporal evolution of the PAPT (and DMAB) Raman spectra for big (i.e. $>30 \mu \mathrm{m}$ ) droplets aged overnight, irradiated at $1.6 \mathrm{~mW} / \mu \mathrm{m}^{2}$. 